\title{
METEORIC IONS IN PLANETARY IONOSPHERES
}

\author{
W. D. Pesnell' and J. M. Grebowsky² \\ 'Nomad Research Inc., 2804 Nomad Court, Bowie, MD 20716, USA \\ ${ }^{2}$ NASA Goddard Space Flight Center, Laboratory for Extraterrestrial Physics, Greenbelt, MD 20771, USA
}

\begin{abstract}
Solar system debris, in the form of meteoroids, impacts every planet. The flux, relative composition and speed of the debris at each planet depends on the planet's size and location in the solar system. Ablation in the atmosphere evaporates the meteoric material and leaves behind metal atoms. During the ablation process metallic ions are formed by impact ionization. For small inner solar system planets, including Earth, this source of ionization is typically small compared to either photoionization or charge exchange with ambient molecular ions. For Earth, the atmosphere above the main deposition region absorbs the spectral lines capable of ionizing the major metallic atoms ( $\mathrm{Fe}$ and $\mathrm{Mg}$ ) so that charge exchange with ambient ions is the dominant source. Within the carbon dioxide atmosphere of Mars (and possibly Venus), photoionization is important in determining the ion density. For a heavy planet like Jupiter, far from the sun, impact ionization of ablated neutral atoms by impacts with molecules becomes a prominent source of ionization due to the gravitational acceleration to high incident speeds. We will describe the processes and location and extent of metal ion layers for Mars, Earth and Jupiter, concentrating on flagging the uncertainties in the models at the present time. This is an important problem, because low altitude ionosphere layers for the planets, particularly at night, probably consist predominantly of metallic ions. Comparisons with Earth will be used to illustrate the differing processes in the three planetary atmospheres.
\end{abstract}

\section{BACKGROUND}

Metallic ions are important constituents of the Earth's lower ionosphere. At night, when photoionization of ambient atmospheric molecules is absent, they are typically the dominant ions in the $E$ region and can contribute to the production of large hydrated molecules in the lower $E$ and $D$-regions. Their 
dominance arises because of the long lifetimes of atomic metal ions and the short chemical lifetimes of the ambient ionosphere molecular ions $\left(\mathrm{O}_{2}{ }^{+}\right.$and $\left.\mathrm{NO}^{+}\right)$. Even during daylight, metal ions are often the dominant ion species. Convergent neutral wind drag forces pile up metal ions in narrow "sporadic E" layers and the local ionospheric molecular ions are rapidly lost due to enhanced dissociative recombination rates associated with the layered electron densities (Narcisi, 1971). There are other important roles the metals may play in the physics of the Earth's near geospace environment. For example, the metal ion layers at night are sometimes the only electric current carrying channels and hence will be a factor in ionospheric electrodynamics. The meteoroid deposited metals, as nucleation agents, may also be involved in the production of noctilucent clouds. Further, the metal ions are excellent tracers of ionospheric dynamics due to their long lifetimes (e.g., Fesen and Hayes, 1982; Kumar and Hanson, 1980; Grebowsky and Pharo, 1985).

Since the first detection of metal ion layers in the terrestrial atmosphere by sounding rocket ion spectrometer measurements, they have been recognized to be the result of ablated material deposited by a continual influx of interplanetary particles (Istomin, 1963). Such meteoroid infalls take place onto all planetary-sized bodies in the solar system. The ablation of atoms from these infalling particles in all atmosphere-laden planets will lead to the production of ionospheric layers. The resulting ionospheric signatures will vary from planet-to-planet depending on atmospheric composition and pressure, the magnitude of gravitational attraction and location in the solar system. These differences in planetary environments provide unique investigative platforms for separating the physical processes underlying the production of meteor ion layers. These processes will be described in this paper by concentrating on the three planets, Mars, Earth and Jupiter, which have contrasting atmospheric structures and gravitational fields.

\section{LAYERS IN PLANETARY ATMOSPHERES}

The only planet for which we have direct observations of the details of metal layers (both ions and some of the neutral species) is Earth. Sounding rockets carrying ion mass spectrometers provide detailed snapshots of the meteoric ion distributions even when they are minor ionospheric species. Ground based remote sensing experiments, such as ionosondes and Incoherent Scatter Radars, can routinely sample meteoric effects. In addition to detecting individual meteor trails, the radio techniques can track the development of overhead sporadic-E layers, of which metallic ions are a major component, and lidars can follow the overhead distribution of neutral $(\mathrm{Na}, \mathrm{K}, \mathrm{Ca})$ and ion species $\left(\mathrm{Ca}^{+}, \mathrm{Fe}^{+}\right)$. Instruments do not exist that are capable of measuring in situ the neutral metal species in the midst of the much denser ambient atmosphere species.

For other planets we have neither in situ measurements of the low altitude ion layers nor remote sensing measurements from the planetary surfaces. Instrument-bearing probes dropped through the atmospheres of Mars, Venus and Jupiter did not make ion measurements nor were they capable of detecting the small concentrations of the neutral metallic compounds. An understanding of the metal ion layers can only be developed by using theoretical models in conjunction with planetary spacecraft radio occultation measurements of the low-altitude electron density distributions. Examples of electron density, radio 
occultation observations for Mars and Jupiter are shown in Figure 2. There appear to be ledges or enhancements in the Martian electron density profiles (between 50 and $90 \mathrm{~km}$ ) below the main ionospheric peak, but the signature is not very prominent and other published low altitude Mars profiles (e.g., see Viking profiles in Haider, 1997) do not show similar structures. However, the Jovian observations do reveal prominent narrow peaks at low altitudes suggestive of terrestrial sporadic E layers structures. Venus also at times has low altitude layers (e.g. see the series of occultation profiles in Kliore, 1979) below the main ambient ionosphere layer, as do Neptune (Lyons, 1995) and Saturn (Kliore et al., 1980). It is probable that metallic ions are responsible for these structures, but in lieu of better observations, theoretical models must be undertaken to explain the radio occultation measurements.

\section{MICROMETEOROID INPUTS}

The degree of ablation and the amount of ionization left in the ionosphere of an atmosphere-laden planet depends on the mass and velocity distributions of the solid particles in the vicinity of the planet and on the speeds with which they impact the atmosphere. Flux-mass distribution models are firmly established for the vicinity of Earth (Love and Brownley, 1993; Dohnanyi, 1972) but measurements at the other planets are sparse, so to a first approximation the Earth distributions are applied adjusting the net flux to each planet's environment. Masses between $10^{-7}$ and $10^{-6} \mathrm{~g}$ contribute the maximum mass flux deposition on Earth. Meteoroid velocity distributions are not well quantified for any of the planets, so one incident velocity is typically used in models, corresponding to the expected average infalling speed for a planet. The maximum entry speed for a planet corresponds to the incidence of a body orbiting the sun in a retrograde parabolic orbit. Particles in open orbits about the sun, i.e., of extra-solar system origin, make a non-significant contribution to the mass input. The minimum speed is the planetary escape velocity. Hence, for Earth the range of speeds is $11-72 \mathrm{~km} / \mathrm{s}$, for Mars it is $5-58 \mathrm{~km} / \mathrm{s}$ and for Jupiter it is $60-69 \mathrm{~km} / \mathrm{s}$. The average speed measured at Earth is $\sim 20 \mathrm{~km} / \mathrm{s}$. Extrapolating the Earth's speed within its range to Mars yields an average incident Mars speed of $-10 \mathrm{~km} / \mathrm{s}$. At Jupiter the range of variation is not significant as will be shown below in Figure 4. There is also a difference in incidence flux from planet-to-planet due to gravitational focusing. The focused flux is equal to $S U\left[1+\left(v_{\text {escape }} / U\right)^{2}\right]$ where $S$ and $U$ are the meteoroid spatial density and average speed far from the planet (Öpik, 1958). Using $S$ and $U$ measured near Earth with observed inverse dependence of density on distance from the sun $R$ and scaling speed by Keplerian dependence on $1 / R^{1 / 2}$ yields an enhancement of fluxes at Jupiter of $\sim 4 \times$ that at Earth and a reduction at Mars of $\sim 0.4 \times$. The exact flux depends on the velocity distributions of the particles, which are poorly known, so the focusing factor is approximately taken as equivalent to that at Earth. Finally, it should be noted that in addition to the continual population of meteoroids that impact each planet - the so-called sporadic population -there are also short duration meteor showers with distinctly different population characteristics. Of these, the fast streams, with speeds approaching the upper limit entry speed for a given planet, could leave their own ionospheric signatures behind. 


\section{ABLATION AND OTHER ATMOSPHERIC INTERACTIONS}

Averaged thermal and physical parameters of meteoroids used in modeling terrestrial ablation processes are typically based on the assumption of stony chondritic composition (i.e., mostly $\mathrm{Mg}, \mathrm{Si}$, and $\mathrm{Fe}$ ). As one moves to the outer planets far from the asteroid belt cometary particles become more important and the average density of the meteoroids is lowered. Fragmentation of particles upon atmospheric impact is not considered important for the micron sized particles that are the dominant source of deposited metal atoms for these the maximum dynamic pressure does not exceed the break-up strength. Further it has often been assumed that all ablation products are atomic, although an arbitrary reduction in net ablation efficiency has been assumed in previous terrestrial studies (e.g., McNeil et al., 1996) to better reproduce measured terrestrial metallic ion concentrations. This implicitly could reflect the effect of recondensation of ablated vapor to form large particles which gravitationally drop out of the atmosphere. A more rigorous parameterization of this effect has been employed for the outer planets (e.g., Moses and Blass, 2000). However, chemical reaction of the vapor metals with the atmosphere might be so effective that the recondensation process might be of minor importance. Until better quantification of these processes and more refined measurements of the interplanetary particle distribution functions are made, models should be considered as providing an order of magnitude estimate of the atmospheric consequences rather than as precise predictions.

The equations used in the current study to compute the material deposited by the ablation process are described in Pesnell and Grebowsky, 2000. They include: the acceleration equation relating the reduction in speed to atmospheric drag; the ablation equation describing mass loss from the meteoroid by evaporation, heating and sputtering (i.e., direct particle collisional ejection from the surface); and the heat equation relating the bulk heating of the meteoroid to frictional heating, radiation loss and evaporative cooling. Details of the computational scheme for applying these equations to model ionization effects can be found in Pesnell and Grebowsky (2000).

Figure 3 (left) depicts atmospheric pressure models for Mars, Earth and Jupiter contrasting the differing atmospheric environments of the three planets. A rough estimate of the position of the main metal ion layer from planet to planet can be made by considering that the point of maximum ablation of a meteoroid occurs when it has intercepted a mass of atmospheric gas equal to its own mass. It is near this region where the meteoroid is most rapidly decelerated. On the right of Figure 2, the column mass density is plotted for each of the planets. A vertical line is drawn which passes through the Earth curve at the altitude where the typical maximum in meteoric ionization has been observed. The intersection with the Jovian curve shows that the metal layer at Jupiter will be above $300 \mathrm{~km}$ (referenced to the 1 bar level) and at Mars it will be near $85 \mathrm{~km}$. These are consistent with the observations in Figure 2 taking into consideration that the exact locations of the ion layer peak will also depend on diffusion and chemical processes.

The neutral atom and ion deposition resulting from a rigorous solution of the meteoroid ablation equations for conditions appropriate to Mars, Earth and Jupiter are plotted in Figure 4. Only magnesium is 
treated here for simplicity. The calculations were made for two incident velocities for each planet - the nominal entrance speed of the sporadic meteor population and a speed near the upper planetary meteoroid speed limit, which would be applicable to a retrograde meteor shower. The ion production rate shown in Figure 4 corresponds to the immediate ionization of the fast moving neutral $\mathrm{Mg}$ by impact ionization with an atmospheric molecule. The ionization probability for impact ionization varies as $\sim v^{3.5}$ where $v$ is the impact speed. The local production of ionization is most significant for high speed shower streams on Earth and Mars, but on Jupiter it is always comparable to the neutral atom deposition rate and the ionospheric effect of a high speed meteor shower would not be clearly discernable from that of the average particle influx.

In addition to the direct deposition of ionization shown in Figure 4, there are other processes by which the neutral metals can be ionized. Indeed the different ionization processes vary in importance from one planet to another. On Earth the most dominant ionization source for metal ions is charge exchange of the ablated neutrals in the lower ionosphere with the ambient species, $\mathrm{NO}^{+}$and $\mathrm{O}_{2}{ }^{+}$. Photoionization is not significant due to the absorption by the atmospheric oxygen of the metal ionizing solar UV lines. For Mars, on the other hand, lower ionospheric densities plus a $\mathrm{CO}_{2}$ atmosphere that allows the penetration of metal ionizing radiation to the ablation altitude, photoionization dominates as the ionization source (Pesnell and Grebowsky, 2000). At Jupiter, the incoming speeds of the meteoroids are so rapid that the metal ion production is dominated by impact ionization of ablated fast moving atoms with the atmosphere (Kim et al., 2000).

\section{PLANETARY LAYERS}

Using the magnesium deposition altitude profiles depicted in Figure 4, full ion-neutral chemical schemes including vertical diffusion have been employed to model the vertical $\mathrm{Mg}^{+}$ion density distributions for the three planets, which have different neutral atmosphere compositions. The calculations for all planets include the effects of photoionization and charge exchange with average, modeled ambient molecular ion distributions, even though these processes do not play a significant role for all of the planets. The chemical chains employed are long and comprehensive. For Mars the magnesium reactions are the same as those for Earth (Pesnell and Grebowsky, 2000), but of course the relative importance of each chemical path varies with atmospheric composition. For Jupiter a complete chemistry chain for the hydrogen/hydrocarbon atmosphere with the best available or estimated rates was compiled by Kim et al., 2000. The biggest uncertainty in the metal ion chemistry is the importance of three-body adduct reactions as loss paths for the ions. The best estimates were made of the rates of unmeasured chemical reactions.

The resulting $\mathrm{Mg}^{+}$profiles are shown in Figure 5. $\mathrm{Fe}^{+}$is also depicted for Jupiter along with the major, modeled ambient ion species. Fe along with $\mathrm{Mg}$ are the dominant meteoroid metal constituents. The difference in the distribution of the two metal ion species on Jupiter is due to the differing reactions of the two metals with hydrocarbons (as discussed in Kim et al. (2000). The chemistry on Mars and Earth for these two species does not lead to any such significant differences in their altitude distributions. In general one expects the amounts of $\mathrm{Mg}^{+}$and $\mathrm{Fe}^{+}$to be similar. Carbon dioxide, although a minor species on Earth, plays a 
role as evident in the production of $\mathrm{MgCO}_{3}$, which appears at both Earth and Mars at low altitudes although it is only the dominant $\mathrm{Mg}$ species on Mars. It seems to be common to all the models that metallic ion densities generally lie in the range of $10^{3}-10^{4} \mathrm{~cm}^{-3}$. For Earth and Jupiter, which have intrinsic magnetic fields, electric fields and neutral wind shears can produce variability in the peaks so that the simple time independent calculations employed here should be interpreted as a first step to fully understanding the metallic ion distributions. Similar magnitudes are predicted for the concentration within metallic ion layers on Saturn (Moses and Bass, 2000) and Neptune (Lyons, 1985) which are consistent with radio occultation layer magnitudes observed at those planets.

\section{CONCLUSIONS}

While the ablation of meteoroids in the various planetary atmospheres is similar, the formation of metallic ions and molecular components is significantly different. Still, models, such as those for Earth, Mars and Jupiter that were discussed in this paper, all predict strong metal ion layers with peak concentrations comparable or exceeding the typical low altitude ambient ionospheric densities on the nightside of the planets. This has been directly measured on Earth, but for the other planets only electron density profiles are available. The lowest layers observed at Jupiter at night seem to be consistent with a metal ion interpretation. The results at Mars are still ambiguous - modeling indicates that a metal ion layer similar to Earth should exist, but the few published low altitude electron density profiles do not show the expected prominent layer below $100 \mathrm{~km}$. Either the model is overestimating the concentrations or the low density threshold of the occultation measurements is not sufficient to clearly resolve the low altitude layers. A similar analysis is being undertaken for Venus, which like Mars has a $\mathrm{CO}_{2}$ atmosphere but a different minor neutral species atmosphere composition and a very different rotational period, possibly leading to strong local time effects on the metal ions. To continue to advance our understanding we need to: further refine the distribution of incident particle properties from planet to planet; quantify the importance of nucleation and condensation; resolve the local time dependence of chemical reactions; take into account atmospheric dynamics and electromagnetic fields; and resolve the effectiveness of adduct reactions

\section{REFERENCES}

Dohnanyi, J. S., Interplanetary objects in review: statistics of their masses and dynamics, Icarus, 17, 1-48, 1972. Fesen, C. G., and P. H. Hays, $\mathrm{Mg}^{+}$morphology from visual airglow experiment observations, J. Geophys. Res., 87, 9217-9223, 1982.

Grebowsky, J. M., and M. W. Pharo III, The source of midlatitude metallic ions at F-region altitudes, Planet. Space Sci. 33, 807-815, 1985.

Grebowsky, J. M., and W. D. Pesnell, Meteor showers: modeled and measured effects in the ionosphere. presented at Reno, NV, AlAA, paper 99-0503, 1999.

Haider, S. A., Chemistry of the nightside ionosphere of Mars, J. Geophys. Res., 102, 407-416, 1997. 
Hinson, D. P., D. T. Twicken, and E. T. Karayel, Jupiter's ionosphere: new results from Voyager 2 radio occultation measurements, J. Geophys. Res., 103, 9505-9520, 1998.

Istomin, V. G., Ions of extra-terrestrial origin in the Earth atmosphere, Space Res.. 3, 209-220, 1963.

Kim, Y. J., W. D. Pesnell, J. M. Grebowsky, and J. L. Fox, Meteoric ions in the ionosphere of Jupiter, Icarus, 2000.

Kliore, A., G. F. Lindal, I. R. Patel, D. N. Sweetnam, and H. B. Hotz, Vertical structure of the ionosphere and upper neutral atmosphere of Saturn from the Pioneer radio occultation, Science, 207, 446-449, 1980.

Kliore, A. J., I. R. Patel, A. F. Nagy, T. E. Cravens, and T. I. Gombosi, Initial observations of the nightside of Venus from Pioneer Venus Orbiter radio occultations, Science, 205, 99-102, 1979.

Krankowsky, D., F. Anold, H. Wieder, and J. Kissel, The elemental and isotopic abundance of metallic ions in the lower E-region as measured by a cryogenically pumped quadrupole mass spectrometer, Int. J. of Mass Spectrom. Ion Phys., 8, 379-390, 1972.

Kumar, S., and W. B. Hanson, The morphology of metallic ions in the upper atmosphere, J. Geophys. Res., 85, 67836801, 1980.

Lebedinets, V. N., A. V. Manochina, and V. B. Shushkova, Interaction of the lower thermosphere with the solid component of the interplanetary medium, Planet. Space Sci., 21, 1317-1112, 1973.

Love, S. G., and D. Brownlee, A direct measurement of the terrestrial accretion rate of cosmic dust, Science, 262, 550553, 1993.

Lyons, J. R., Metal ions in the atmosphere of Neptune, Science, 267, 648-651, 1995.

McNeil, W. J., S. T. Lai, and E. Murad, A model for meteoric magnesium in the ionosphere, J. Geophys. Res., 101, 5251-5259, 1996.

Moses, J. I., Meteoroid ablation in Neptune's atmosphere, Icarus, 99, 368-383, 1992.

Moses, J. I., and S. F. Bass, The effects of external material on the chemistry and structure of Saturn's ionosphere, $J$. Geophys. Res., 105, 6983-6996, 2000.

Narcisi, R. S., Composition Studies Of The Lower Ionosphere, in Physics of the Upper Atmosphere, ed. F. Verniani, pp. 11-59, Editrice Compositore, Bologna, 1971.

Öpik, E. J., Physics of Meteor Flight in the Atmosphere, Interscience, New York, 1958.

Pesnell, W. D., and J. M. Grebowsky, Meteoric magnesium ions in the Martian ionosphere, J. Geophys. Res., 105, 1695-1708, 2000.

Savich, N. A., V. A. Samovol, M. B. Vyshlov, A. S. Samoznaev, A. I. Sidorenko. and D. Ya Shtern, The nighttime ionosphere of Mars from Mars 4 and Mars 5 radio occultation dual-frequency measurements, in Solar Wind Interaction with the Planets Mercury, Venus and Mars, ed. N.F. Ness, NASA SP-397, pp. 41-46, 1976.

Zbinden, P. A., M. A. Hildago, P. Eberhardt, and J. Geiss, Mass spectrometer measurements of the positive ion composition in the D- and E-regions of the ionosphere, Planet. Space. Sci., 23, 1621-1642, 1975. 

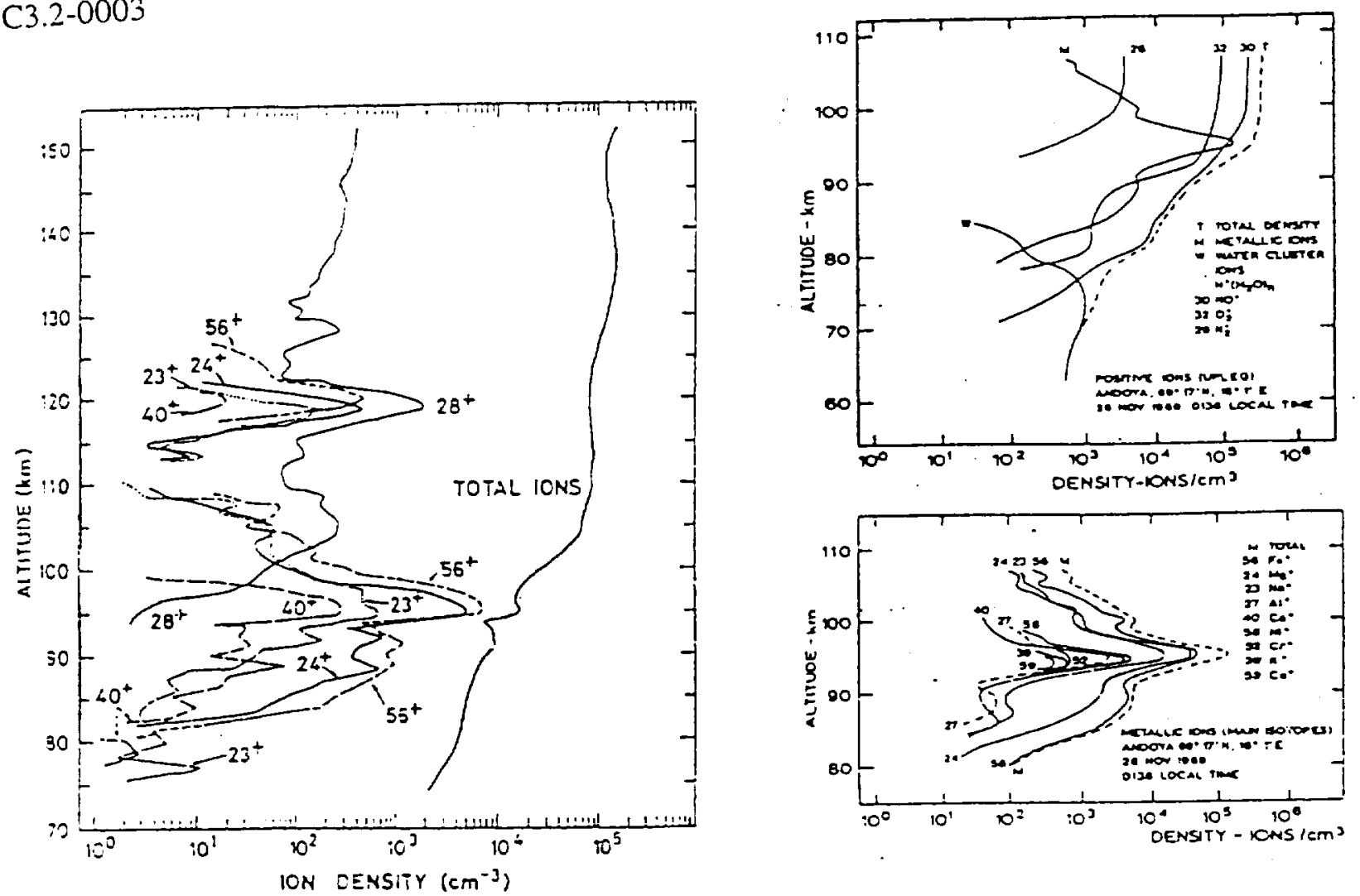

Figure 1. At Earth a persistent metal ion layer is present between 90 and $100 \mathrm{~km}$ with the frequent appearance of multiple layers as seen on the left. Feak densities are between $-10^{3}$ and $-10^{5} \mathrm{~cm}^{-3}$. Fe and $\mathrm{Mg}^{-}$are the dominant meteoric ion species. Data on left is from Zbinden et al. (1975) and on right is from Krankowsky et al. (1972).

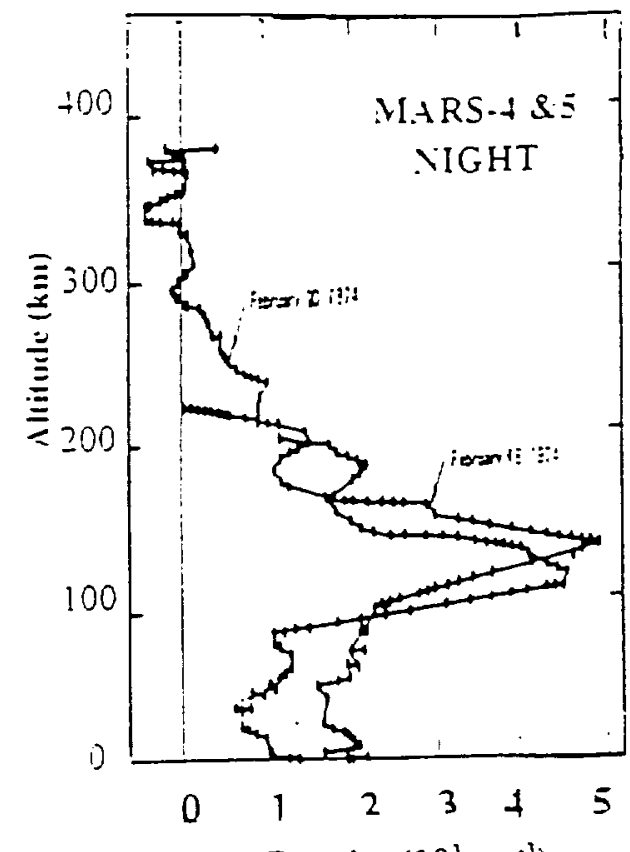

Electron Density $\left(10^{3} \mathrm{~cm}^{\cdot 3}\right)$

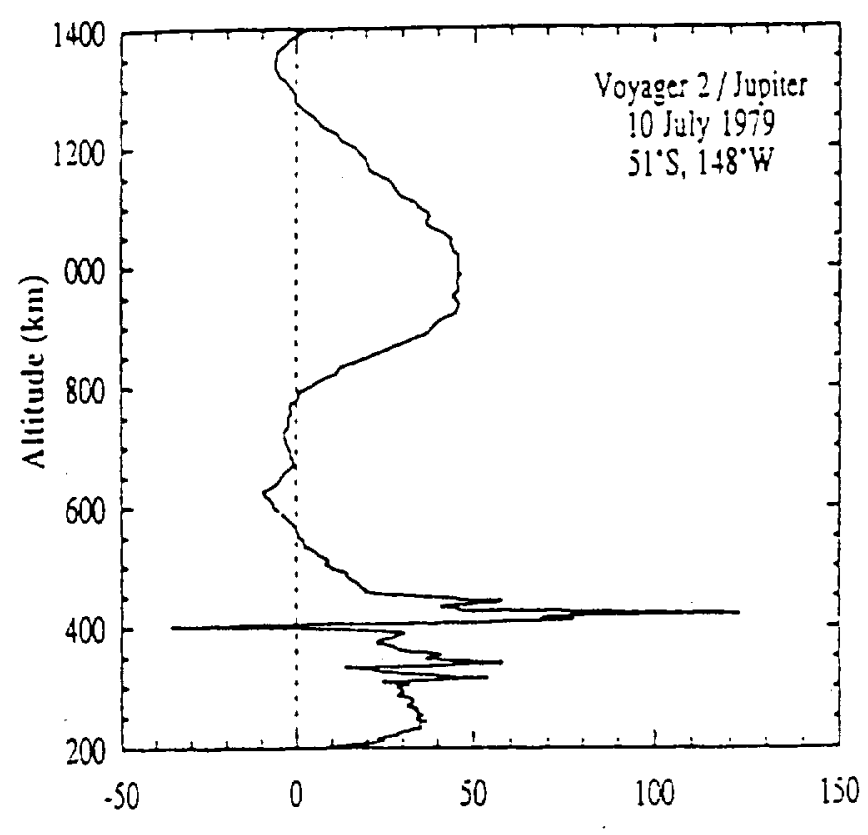

Electron Density $\left(10^{3} \mathrm{~cm}^{-3}\right)$

Figure 2. Radio occultation measurements provide clues for presence or absence of low altitude, metal ion lavers. Observations on left from the USSR Mars 4 and 5 missions (Savich et al., 1976) show no clear layers beiow the main ionospheric peak above $100 \mathrm{~km}$. The Voyager measurements, on the left, at Jupiter do show narow structured low altitude layers (in particular the one near $400 \mathrm{~km}$ - relative to the $1 \mathrm{bar}$ altitude) that are consistent with a meteoric ion layer interpretation (from Hinson et al., i998). 

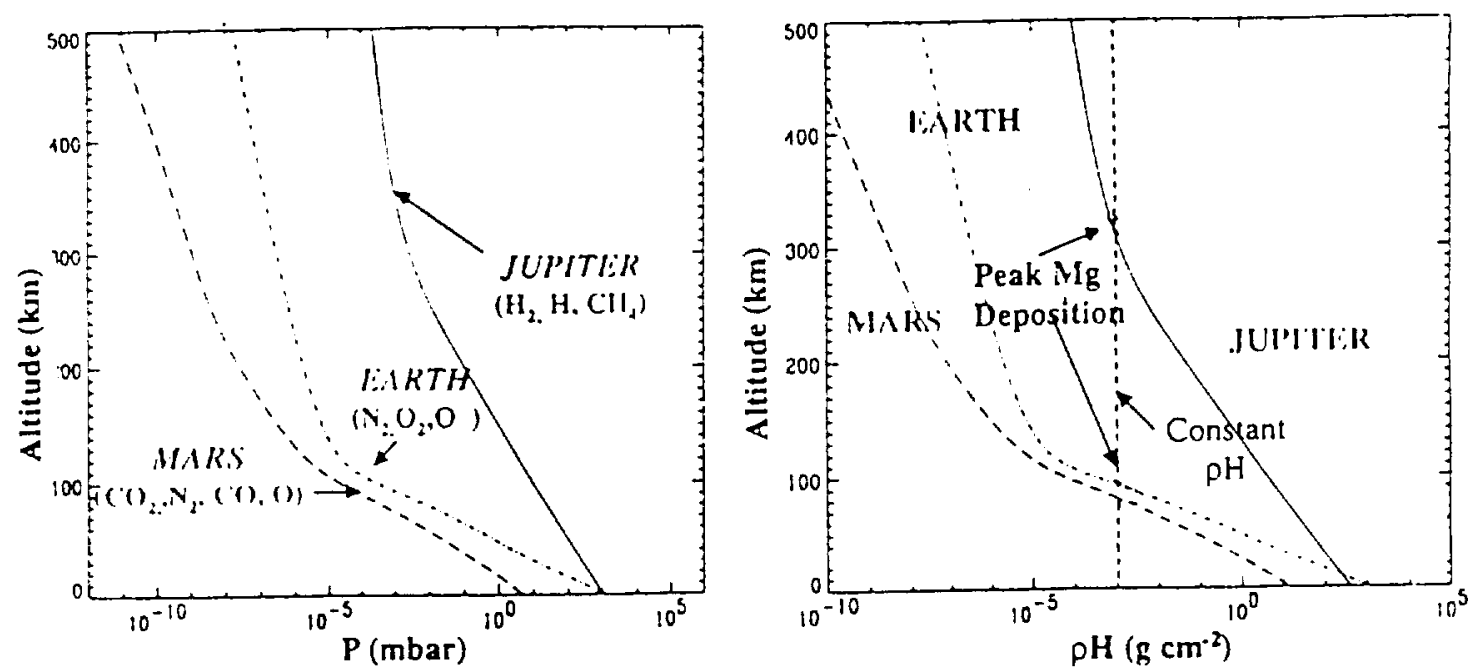

Figure 3. Atmosphere pressure models for major reactive species (left) used in ablation calculations. Peak ablation ( $\mathrm{Mg}$ was species modeled) altitude changes from planet to planet depending on where incoming meteoroids intercept same amount of gas. On right the total mass above each altitude is shown and the vertical line references Juniter and Mars location relative to that at Earth.
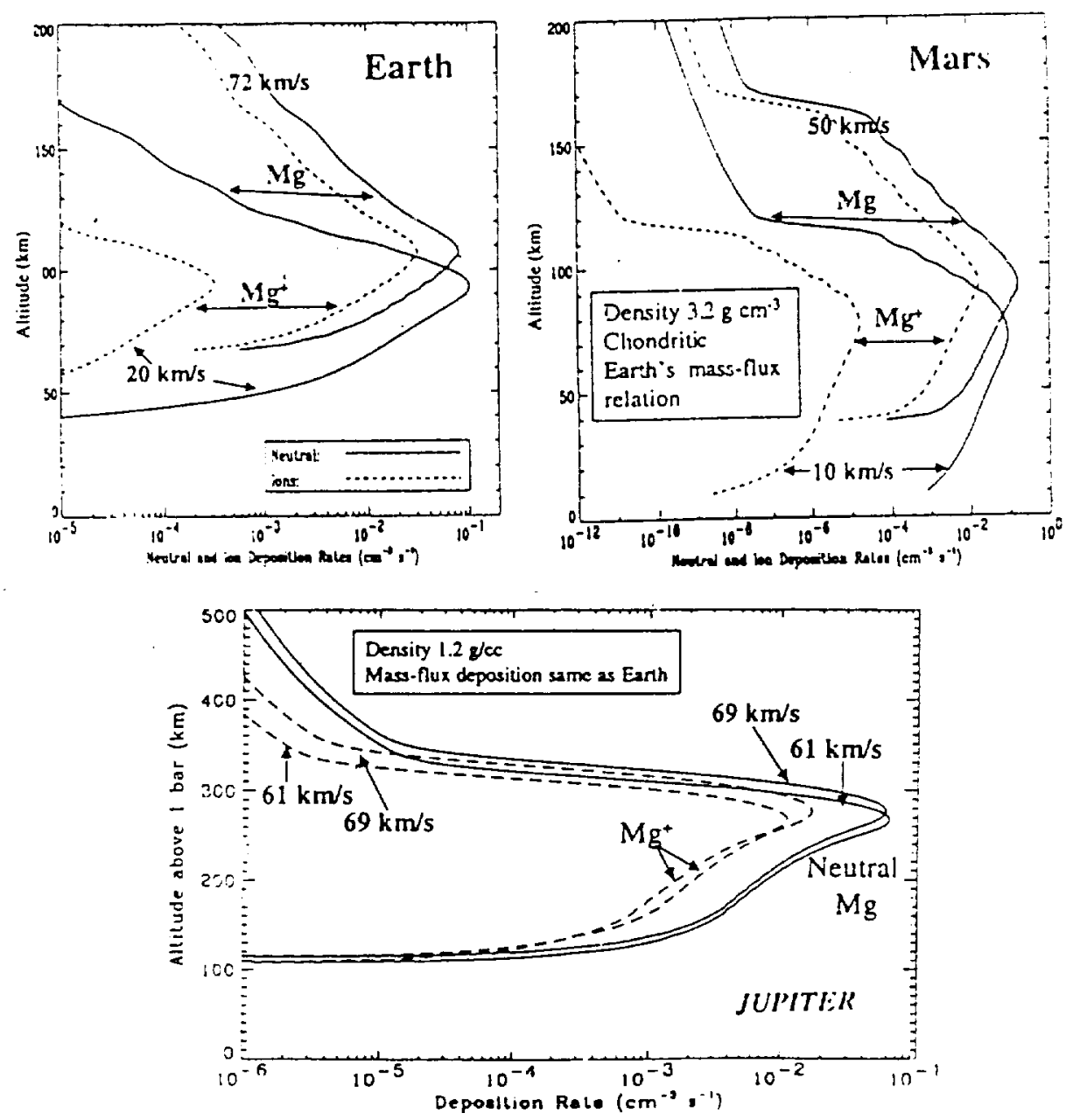

Figure 4. Deposition rates of $\mathrm{Mg}$ from ablation and $\mathrm{Mg}^{+}$from impact ionization of ablated atoms.

For Jupiter there is little change within the range of speeds of incident meteoroids. At Earth and Mars there is a significant increase in the altitudes of the maximum and the production of ionization as the incident speeds increase. The high-speed behavior would be characteristic of some meteor showers. 

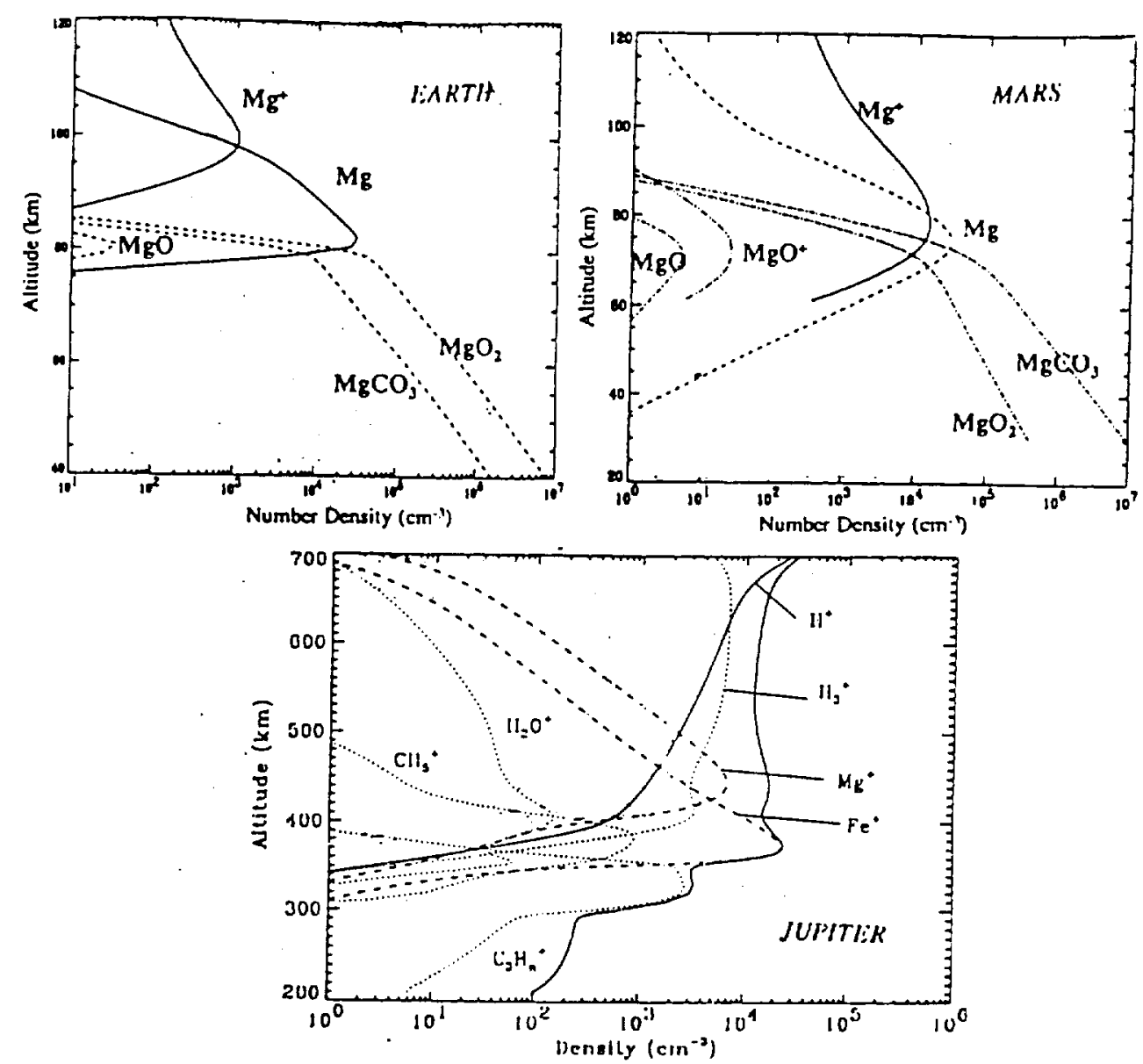

Figure 5. Magnesium ions and neutral species for Earth (Grebowsky and Pesnell, 1999) and Mars (Pesnell and Grebowsky, 2000) show similar properties. Jupiter model results (Kim et al., 2000), which is a complete ionosphere model, sampled at a predawn local time, predicts metallic ions to be dominant below the main ion layer. 